The percentage of methylated cytosine in leukocyte DNA was measured as a marker of global DNA methylation in 775 patients with bladder cancer (cases) and 397 control participants.

Overall, the median percentage of methylated cytosine was significantly lower in cases than in controls $(3.03 \%$ vs $3.18 \%$, interquartile ranges $2.17-3.56 \%$ and $2.46-3.68 \%$, respectively; $P=0.0002)$. All participants were grouped into quartiles by percentage of methylated cytosine; those in the bottom three quartiles had a significantly greater risk of bladder cancer compared with the top quartile $(P \leq 0.0001$ for trend). Global DNA methylation was not associated with age, sex, smoking status, nutrient intake or single nucleotide polymorphisms in genes involved in folate metabolism and DNA methylation.

The authors conclude that the amount of genomic DNA methylation might be a useful phenotypic marker for determining the risk of bladder cancer. Further study of this association in other cancer types is warranted.

Original article Moore LE et al. (2008) Genomic DNA

hypomethylation as a biomarker for bladder cancer susceptibility in the Spanish Bladder Cancer Study:

a case-control study. Lancet Oncol 9: 359-366

\section{Restoration of the penis after accidental amputation during circumcision}

The complication rate associated with male circumcision varies worldwide from $0.1 \%$ to $35 \%$. While most complications are minor (such as infection and bleeding), serious complications do occur. Amputation, which can be caused by entrapment of the penile shaft between the blades of the clamp or by thermal injury when unipolar diathermy is employed, is a particularly severe complication of circumcision. Shaeer has described outcomes of his own surgical technique for penis restoration in 32 patients (aged between 2 months and 6 years) subjected to accidental penile amputation during circumcision.

Briefly, Shaeer's A-Y plasty involved exposure and advancement of the remnant proximal corpora cavernosa and crura to an adequate extent to form the new shaft. To prevent retraction of the penis, a fat flap, formed from the pubic area by an A-shaped incision, was pushed down to fill the gap between the released penis and the pubis. The phallus was covered with dartos muscle flaps and either a full-thickness or split-thickness skin graft.

Surgery resulted in acceptable functional and cosmetic outcomes in all 32 patients, with normal nocturnal erections and penile lengths within normal ranges for the patients' agegroups (mean length $\sim 2 \mathrm{~cm}$ for patients aged $<1$ year and $3.4 \mathrm{~cm}$ for patients aged 1-6 years). Only one patient so far has reached puberty; he reported normal sexual function, and neoglansplasty is planned for this patient. Although long-term, postpubertal outcomes for the other patients remain to be seen, the author recommends his technique as an alternative to neophalloplasty or sex reversal.

Original article Shaeer O (2008) Restoration of the penis following amputation at circumcision: Shaeer's A-Y plasty. J Sex Med 5: 1013-1021

\section{Factors that influence patients' participation in prostate cancer trials}

Clinicians face problems recruiting patients for prostate cancer trials. Studies of the barriers to and facilitators for the recruitment process have been conducted for some types of cancer, but are lacking for prostate cancer. Davison and colleagues studied the factors that influence the decision of Canadian men with prostate cancer to enroll in clinical trials.

The researchers developed a 'factors influencing participation' questionnaire. Participants -122 men with prostate cancer who had never been asked to participate in a clinical trial-completed the questionnaire by stating the influence (graded from 1 'not at all' to 5 'a great deal') that 30 suggested variables would have on their decision whether to enroll.

Important influences were helping future patients with prostate cancer, the impact of study treatment on survival and future quality of life, relationships with specialists and being fully informed about the study. Factor analysis identified three subsets of related items: acceptability items (e.g. patients' assessments of personal risk and benefit) were especially important to newly diagnosed patients; awareness items (recruitment process, 